\title{
Use of dependency and prioritization tools by clinical nurse specialists in palliative care: an exploratory study
}

\author{
Mairéad Bracken, Kathleen McLoughlin, Sinéad McGilloway, Edith McMahon
}

$\mathrm{R}$ ecent years have seen an increasing need for professional palliative care and support to be provided to patients in the community in the UK (Sutherland and Midgley, 2011). This is due, in large part, to the growth in the elderly population (Manton et al, 1993; Parker and Thorslund, 2007; Grant et al, 2009), shorter hospital stays (Byrne et al, 2006), changes in health service policy (Payne et al, 1999), and a growing recognition of the desire of many palliative care patients to die at home (Yao et al, 2007).

Clinical nurse specialists (CNSs) who deliver specialist palliative care (SPC) in the community play a key part in caring for patients and supporting families at the end of life. CNSs visit and treat patients with advanced progressive life-limiting illnesses whose prognosis would typically be less than 6 months. SPC teams aim to improve the quality of life of these patients (Gibbs et al, 2002) and have also been shown to reduce the number of inpatient hospital days and the period spent by patients in out-of-home services (Hearn and Higginson, 1998). The CNS role comprises five key areas-clinical skills, teaching, consultation, leadership, and research-although an important additional component of the clinical aspects involves spending time with patients and their families and helping them come to terms with a range of complex emotional problems (Skilbeck and Payne, 2003; Aitken, 2009). CNSs also support primary care staff in caring for palliative patients in the community by offering advice and education on holistic symptom assessment and management. In the Republic of Ireland (ROI), the patient population for CNSs working in SPC generally comprises patients with both malignant and non-malignant disease.

However, there are several difficulties in how palliative care services are perceived by the public and professionals that affect when and how they are accessed (Ahmed et al, 2004; Fadul et al, 2009; Hirai et al, 2011). These include the heterogeneity in the palliative care services available across different areas, resistance on the

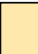
Abstract
Aims: The principal aim was to assess the utility of three needs assessment/dependency tools for use in community-based palliative care services. Specific objectives were to assess a sample of patients receiving specialist palliative care community nursing using these tools, to assess the predictive ability of each tool, and to explore the utility of prioritizing and measuring patient dependency from a clinical nurse specialist (CNS) perspective. Method: In phase I, 22 community-based CNSs completed the Vale prioritization tool for all patients visited during a 3-month period $(n=162)$. They also completed either the Graves and Payne (2007) or the Birch et al (1997) dependency tool after each visit. In phase 2 a focus group $(n=8)$ and two one-to-one interviews with CNS participants explored the perceived utility of all three tools. Results: The Vale prioritization tool appeared to be the most useful for prioritizing patient need and managing workload. Statistical analysis highlighted minimal differences between the two dependency tools, neither of which predicted length of visit. Three themes were identified from phase 2: difficulties with routine administration, points of divergence between the two dependency tools, and workload concerns. Conclusion: While the Vale prioritization tool emerged as the most useful, the findings raise questions about the overall utility and practical application of these kinds of tools with community-based palliative care patients. Further research is needed to identify/develop, adapt, and evaluate appropriate, setting-specific dependency tools for use with this population.

Key words: Dependency Prioritization Tools Clinical nurse specialists Workload Palliative care
\end{abstract}

part of some professionals to refer patients to palliative care, and reluctance among many patients and carers to be referred for palliative care owing to a misunderstanding about what it offers (Ahmed et al, 2004). Arguably, these challenges may affect the CNS role and prevent it being effectively fulfilled.

The CNS workload is generally perceived to be considerable and also appears to have increased in recent years (Dark et al, 2011), particularly in view of the expanding provision of palliative care services to people with non-malignant as well as malignant disease (Fisher, 2006; Yang et al, 2011). This may, in turn, lead to concerns about
MB is Research Assistant, and $\mathbf{S M}$ is Senior Lecturer and Director of the Mental Health and Social Research Unit, Department of

Psychology, National University of Ireland (NUI) Maynooth, Ireland; $\mathbf{K M}$ is Head of Education, Research and Professional Development, and EM is Clinical Nurse Manager, Milford Care Centre, Castletroy, Limerick, County Limerick, Ireland

Correspondence to: Sinéad McGilloway sinead.mcgilloway@ nuim.ie 
'...efficient and
effective
workforce
planning in
community
nursing is
crucial for
ensuring
adequate,
timely service
provision for
patients and
their families...'

occupational stress. For example, Rauhala et al (2007) reported a linear trend between increasing workload and increasing sick leave in a nursing population. Despite these concerns, research in this area is limited and little is known about the working practices of CNSs and how they manage their complex caseloads (Lewis and Pontin, 2008). Arguably, efficient and effective workforce planning in community nursing is crucial for ensuring adequate, timely service provision for patients and their families while also helping to alleviate the potentially onerous workloads of community-based nurses.

Several tools and systems have been developed for routine administration in health-care settings to help nurses better estimate and prioritize patient needs. These include the Leeds Elderly Assessment Dependency Screening Tool (Slade et al, 2006), the Pediatric Early Warning Tool (Haines et al, 2006), and the Jones Dependency Tool (Crouch and Williams, 2006). However, these all have their limitations (Dark et al, 2011). For example, many can only be used in the settings in which they were designed, and their validity has also been called into question. The evidence in relation to caseload management and dependency tools in community professional populations is also limited (Kolehmainen et al, 2010).

An extensive review of the literature was undertaken prior to this study to identify any empirical patient dependency and workload assessment tools for use by CNSs when delivering community-based SPC. The UK Hospice at Home Forum was also consulted. As a result, three tools were identified in the published literature and a further tool was identified through personal correspondence. One of these-the Community Client Need Classification System (Byrne et al, 2006)—was deemed to be unsuitable for use by CNSs working in the current setting as it was designed to be used by public health nurses. The remaining three-a 'prioritization tool' and two dependency tools-were subsequently reviewed by the home care team of the palliative care centre in which this study was conducted. Collectively, it was agreed that all three tools could potentially be used, or adapted for use, in assessing the dependency of community-based palliative care patients in the hospice catchment area.

This exploratory mixed methods study was undertaken to administer these three tools to a sample of patients in receipt of SPC community nursing, to ascertain the predictive ability of each tool (i.e. by comparing patient needs at the time of the initial visit (as assessed by each tool) with the length of time spent with the patient during subsequent visits), and to explore the utility of prioritizing and measuring patient dependency from a CNS perspective. A secondary aim was to briefly assess the extent to which the CNS participants were feeling stressed through overwork.

\section{Design \\ Method}

The study took place in two phases. In phase 1 , 22 community-based CNSs completed the Vale prioritization tool for all patients visited during a 3 -month period $(n=162)$. The nurses also completed either the Graves and Payne (2007) or the Birch et al (1997) dependency tool after each visit (typically 5 visits per patient). Each of these tools is described below, but no information on the reliability or validity of these measures could be located. Thus, it would appear that all are still in the early stages of development. In phase 2 a focus group ( $n=8)$ and two one-to-one interviews with CNS participants were undertaken to explore the perceived utility of all three tools.

\section{Setting}

The study was undertaken in a community-based palliative care centre located in a south-western region of the ROI. It serves three counties with a total population of around 339500; this constitutes approximately $9 \%$ of the total population of the ROI. The catchment area is characterized by an urban-rural mix and diverse levels of population density. The centre manages referrals for over 1000 community palliative care patients and is host to Ireland's first consultant-led multidisciplinary hospice at home team.

\section{Sample/participants}

A convenience sample of 22 CNSs took part in the first phase of the study, 10 of whom also participated in the qualitative interviews in phase 2. All of the CNSs in the palliative care centre team were invited to participate, and all did participate in completing the tools as this was an initiative adopted by the team as a whole. The participants were female with an average age of 41 years (range 21 years). The average number of years working in palliative care was 13 (range 15), while the average number of years working as a CNS was 5 (range 5). All 22 participants were asked to take part in phase 2 of the study and those who were available to take part did so.

The CNSs completed tools on the patients they visited and treated in the study period. The patients presented with various malignant and non-malignant diseases such as motor neurone disease, end-stage chronic obstructive pulmonary 


\section{Table I. Sample of Graves and Payne (2007) dependency tool items}

\begin{tabular}{|c|c|c|c|c|}
\hline Visit/date: & Level I & Level 2 & Level 3 & Level 4 \\
\hline \multicolumn{5}{|c|}{ Patient's physical dependency score } \\
\hline \multicolumn{5}{|c|}{ Patient's psychological dependency score } \\
\hline \multicolumn{5}{|c|}{ Carer's physical dependency score } \\
\hline \multicolumn{5}{|c|}{ Carer's psychological dependency score } \\
\hline Travel time & $<30$ mins & 30 mins & $30-60$ mins & $>1$ hour \\
\hline Length of visit & $<30$ mins & $30-60$ mins & $>1$ hour & $\geq 2$ hours \\
\hline No of phone calls & $<2$ & $2-5$ & $5-10$ & $\geq 10$ \\
\hline Total Score & & & & \\
\hline
\end{tabular}

\section{Table 2. Sample of Birch et al (1997) dependency tool items}

\begin{tabular}{l|l|l|l|l|}
\hline $\begin{array}{l}\text { Visit/date: } \\
\text { Physical }\end{array}$ & Level I & Level 2 & Level 3 & Level 4 \\
\hline $\begin{array}{l}\text { Emotional/spiritual } \\
\text { Social }\end{array}$ & & & & \\
\hline Travel time & $<30$ mins & 30 mins & $30-60$ mins & $>1$ hour \\
\hline $\begin{array}{l}\text { Length of visit } \\
\text { No of phone calls }\end{array}$ & $<30$ mins & $30-60$ mins & $>1$ hour & $\geq 2$ hours \\
\hline Total score & $<2$ & $2-5$ & $5-10$ & $\geq 10$ \\
\hline
\end{tabular}

disease (COPD), cardiac failure, renal failure, and Parkinson's disease.

\section{Measures}

Phase 1: Vale prioritization tool

The (unpublished) Vale prioritization tool (personal correspondence) was developed originally with a view to prioritizing cancer patient care, but it was used here to provide a 'dependency profile' of each patient (i.e. prior to administering either dependency tool). It comprises ten dimensions, four of which were excluded from the analysis here as they duplicate the dimensions in the dependency tools (i.e. patients' and carers' physical and psychological needs). The remaining six dimensions were 'prognosis', 'location', 'lives alone', 'resident carer', 'other services', and 'what would happen if need not met'. Dimensions 1, 2, 5 , and 6 are scored from 1-4 in the direction of greatest priority. The remaining two categories require only a 'yes' or 'no' response, for a score of 4 and 1 respectively. A total score on the prioritization tool may be computed by summing the scores assigned to each of the dimensions and so here ranged from 6 to 24 .

Graves and Payne (2007) and Birch et al (1997) dependency tools

Both dependency tools were designed originally with a view to measuring dependency in patients, although the Graves and Payne tool also incorporates an assessment of carer dependency. Thus, its categories relate to the 'physical dependency' and 'psychological dependency' of both patients and their carers (Table 1). The Birch et al tool, on the other hand, focuses on assessing patient dependency only along three key dimensions: 'physical', 'emotional/spiritual', and 'social' (Table 2). Each category in both tools is assigned a score of 1-4 in the direction of increasing dependency. For the purposes of this study, three items were added to each tool to reflect additional factors that the CNS managers considered to be important: an assessment of travel time, number of telephone calls, and length of visit. These were scored in the same way as the above dependency items, so that overall scores ranged from 7 to 28 on the Graves and Payne tool and from 6 to 24 on the Birch et al tool.

The Vale prioritization tool was completed by the CNS participants for all of the patients they visited during an approximate 3 -month period $(n=162)$. It was completed only once for each patient. Participants were then randomly assigned to either the Graves and Payne tool or the Birch et al dependency tool, which they subsequently completed after each patient visit. Each patient received five visits on average during the study period. 


\section{'The evidence \\ in relation to caseload management \\ and \\ dependency \\ tools in \\ community \\ professional \\ populations is \\ limited.9}

Phase 2

Following the completion of phase 1, a topic guide and interview schedule were devised to collect the qualitative data from the focus group $(n=8)$ and interviews $(n=2)$ respectively. Participants were asked about their satisfaction with and experiences of using the tools during the study period, and were also briefly asked their views of their workload. Questions asked included whether they found the tools useful for estimating the time spent with each patient, whether any questions should be added to or removed from the tools, which tool best reflected the reality of their workload, whether the tools captured indirect nursing care, whether they perceived long-term use of such tools to be beneficial, and whether the use of such tools could reduce workload.

An Overwork Stress Spiral (Newton and Waters, 2001) was also administered to all Phase 2 participants $(n=10)$ to provide a brief measure of work-related stress. This part of the study took place owing to anecdotal and research evidence indicating that palliative care staff have a high workload (Fisher, 2006). In addition, if the stress 'spiral' continues, nurses may experience problems in their home life or become ill and, as a result, may choose to leave their job (Schaffer and Norlander, 2009).

\section{Ethical considerations}

The study was conducted in accordance with the ethical codes of conduct of the British Psychological Society and the Psychological Society of Ireland. All of the CNS participants volunteered to take part on the understanding that this was a pilot study. They were given an information sheet and provided written informed consent to take part (and to have the interviews recorded where applicable). No personal patient data were recorded at any time. The focus group and interviews took place in the care centre where the nurses are employed and where they felt most comfortable.

\section{Data analysis}

The quantitative data were analysed using SPSS version 15 with the aim of exploring the relationship between each of the dependency tools and the Vale prioritization tool. Descriptive statistics were generated first, followed by a series of statistical tests.

The qualitative data were recorded, transcribed verbatim, edited for purposes of clarity only, and subjected to a standard thematic analysis (Hayes, 2000) in order to identify and explore key themes and issues relevant to the research questions. The thematic analysis involved categorizing, coding, and classifying pieces of text. The first stage of the analysis involved physically organizing and subdividing the material into main categories. Once an initial set of categories had been established, distinct segments of text (e.g. sentences or phrases) associated with this category were identified. The categories were colour-code mapped so that all data relating to each theme could be identified for further review and reflection. On completion of this process, the data were rigorously examined and a sample of transcripts was also double-coded to allow extraction of and agreement on meaningful and informative themes relevant to the study.

\section{Results}

\section{Phase one: analysis of tools}

In phase $1,57 \%$ of the patients (90/162) were assessed using the Graves and Payne tool; the remaining $43 \%(72 / 162)$ were assessed on the Birch et al tool. Tool usage was unmatched as the tools were distributed to the participants based on treatment area (i.e. the work areas designated to the CNSs). The preliminary results indicated that there was no significant difference $(P>0.05$ here and throughout $)$ in the total scores on the Vale prioritization tool between the patients then assessed using the Graves and Payne tool (mean score on Vale tool 13.95, standard deviation (SD) 3.46) and those assessed using the Birch et al tool (mean 13.66, SD 3.66). This suggests that the two groups had broadly similar profiles.

A one-way between-groups analysis of variance was conducted for each of the two dependency tools to explore any differences between total Vale prioritization scores by length of visit (as categorized into 4 levels: $<30$ minutes, 30-60 minutes, $>1$ hour, and $\geq 2$ hours). No statistically significant differences were found $(\mathrm{F}\{3,144\}=1.10, P=0.35)$. A further correlational analysis showed no relationship between total Vale scores and total dependency tool scores and the duration of each of the first five visits as recorded on each tool.

An analysis of the mean dependency scores calculated for each visit showed that for both groups these tended to remain stable over the first few visits with only a slight increase thereafter. This suggests that although patients were nearing the end of life they were not becoming increasingly dependent. A further Chi squared analysis showed that differences in patient dependency scores by treatment area fell just short of statistical significance $(x 2(6, n=151)=12.05, P=0.06)$. 
Specifically, patients in the north (mean 88.52) and east (mean 92.11) of the region obtained the highest dependency scores overall.

An examination of the relationship between the six different dimensions on the Vale prioritization tool (using scatterplots and crosstabs) revealed only weak associations. Next, a Pearson correlation analysis was conducted between total Vale prioritization score and each of 'lives alone', 'resident carer', and 'other service'. Similar analyses were not undertaken for the other dimensions owing to the absence of any clear relationship in the scatterplots. The results showed strong positive correlations between total score and 'lives alone' ( $\mathrm{r}=0.73, n=154, P<0.0005)$ and 'resident carer' ( $r=0.70, n=154, P<0.0005)$, indicating that those who lived alone and who did not have a resident carer had higher overall levels of dependency. With respect to receipt of 'other services', the results showed a moderate positive correlation between the two variables $(\mathrm{r}=0.58$, $n=155, P<0.0005)$, such that those with fewer services available to them obtained higher total prioritization scores.

Lastly, a standard multiple regression was used to assess the ability of the 'lives alone' and 'other services' variables to predict total scores on the Vale prioritization tool. (The third variable, 'resident carer', was removed from the analyses due to multicollinearity between this variable and 'lives alone'.) These two predictors accounted for almost $80 \%$ of the variance in total score $\left(\mathrm{R}^{2}=0.79\right)$, which was highly significant $(F(2,148)=290.05, P<0.0005)$. Both 'lives alone' $(\mathrm{B}=0.68, P<0.0005)$ and 'other services' $(\mathrm{B}=0.51$, $P<0.0005)$ demonstrated significant effects on the total score.

\section{Phase two: CNS attitudes and views}

Three key themes identified from the thematic analysis were difficulties with routine administration, points of divergence between the dependency tools, and workload concerns. Although the participants agreed that both dependency tools were relatively brief and easy to complete, there was a consensus that neither tool completely captured the length of telephone calls with patients/families, consultations with the multidisciplinary team outside visits, nor the fact that some patients had a dependent carer. For example, from the interviews it emerged that some carers were disabled or ill-in some cases as unwell as or more unwell than the patient themselves. Furthermore, it was agreed that the tools did not adequately cover time spent on indirect nursing care (e.g. liaison with other health professionals):
'There's a huge amount of work done over the phone ... time spent over the phone is another type of care ... there was nowhere to record that.'

'It doesn't capture the indirect nursing care area.'

'Time spent consulting with public health nurses and GPs etc is not accounted for on the tool.'

'They could have a registered carer but that carer could be as unwell as the patient. They could be more dependent.'

Another difficulty related to the observation that patient dependency changes rapidly in SPC. Consequently, a score of 1 on one dependency scale may increase to a score of 3 within a period of hours; this calls into question the reliability and validity of dependency tools in general in the area of SPC:

'Situations change so rapidly in palliative care.'

'The patient changes every day.'

The focus group participants also discussed the possibility that patients may become more dependent because of the CNSs' regular visits. For example, it was suggested that patients may become overly reliant on regular visits and that their dependency scores may be continuously elevated because they feel that they need to be visited:

\section{'Are they dependent because we're going?'}

However, not all of the participants agreed with this view:

'Well I think that's very individual for the patient and the clinical nurse specialist as well. Some people like to feel dependent, you know, like to feel like they're the only one.'

The second theme related to perceived points of difference between the dependency tools. Overall, it appeared that the Graves and Payne tool was preferred. The participants felt that this tool was particularly useful because the separate recording of patient and carer scores allowed more information to be gathered. The recording of carer dependency was considered particularly important owing to the carer illness and dependency already mentioned:

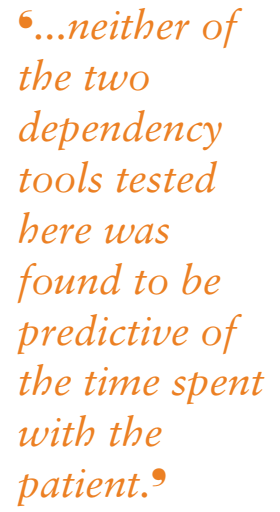

...neither of

the two

dependency

tools tested

here was

found to be

predictive of

the time spent

patient.9 


\section{- An analysis of \\ the mean \\ dependency \\ scores ... \\ showed that \\ for both \\ groups these \\ tended to \\ remain stable \\ over the first \\ few visits with \\ only a slight \\ increase \\ thereafter. 9}

\begin{abstract}
'You get to provide more information [in the Graves and Payne tool].'
\end{abstract}

'This [Graves and Payne tool] is more specific to patients and carers.'

However, the inclusion of a spiritual/emotional category in the Birch et al tool was particularly appealing to some participants:

'There's spiritual in this one [Birch et al tool].'

Therefore, these results highlight that neither tool as it was used in the current study is ideal for measuring all aspects of patient and carer dependency levels.

The final theme that emerged from the analysis related to the high levels of stress and the heavy workloads among the participants:

'Everyone knows that there's a lot of work. There's high dependency of patients-there's a lot of stress within the team. Everyone's complaining about that and making it worse and it fuels the fire.'

It became evident in the qualitative analysis that the participants felt under strain in their current occupational role. Further analysis is needed to establish whether this attitude is predominant within the service. The above comments were further supported and amplified by responses to the Work Stress Spiral, on which most of the interviewees marked their position around the midpoint, centring around terms such as 'decreased enthusiasm', 'work spilling over into days off-not "switching off"', 'becoming dissatisfied with one's own performance, and "not doing anything well",.

\section{Discussion}

Recent research has shown that the introduction of clinical assessment tools may result in important enhancements to care provision at individual service level, increased communication, improved assessment of patient needs, and enhanced identification of triggers for the need to change care plans or for referral (Rawlings et al, 2011). The principal aim of the research reported here was to assess, using mixed methods, the use of three tools designed to provide an assessment of need/dependency among community-based palliative care patients. Arguably, any attempts to quantify patient need and therefore to measure the workload of community nurses poses certain challenges in the absence of a holistic assessment of patients prior to discharge from hospital. This may be further compounded by a lack of ongoing monitoring and review, and anecdotal evidence suggests further that there are many aspects of nurses' workloads that are not recognized or formally acknowledged by their employers.

Byrne et al (2007) suggest that some tools may prove useful in predicting the amount of time required by patients, and it was thought that the tools used in this study may have some predictive ability in this respect. However, neither of the two dependency tools tested here was found to be predictive of the time spent with the patient. Overall, the Vale prioritization tool appeared to provide the most accurate assessment of patient need/dependency and CNS workload. The extent to which patients live alone and the extent to which they avail of other services appear to be particularly important factors. This suggests that a simple recording of this kind of information in the patient's file would provide a useful insight into overall dependency, perhaps without the need to use a structured dependency tool at all. Alternatively, it may be possible to consider adding other categories of this kind to the tools used here in order to ascertain whether this improves their overall predictive ability. The nurses' preference for the Graves and Payne tool, which included an assessment of carer dependency, also reflects the uniquely holistic philosophy of palliative care, with its focus on both patients and family carers.

Brady et al (2007) argue that, while several workload measurement tools may be used to record direct nursing care, these do not take account of indirect nursing care. Similarly, the tools used in the current study failed to take indirect nursing care into account, and this emerged as an important finding during the qualitative phase of the study. Although the number of telephone calls was included as an extra variable (for both tools), the duration of calls could not be recorded and this was considered an important omission by participants. Newbury et al (2008) examined a range of activities within clinical contact time and found that, on average, $6.2 \%$ and $6.4 \%$ of the work schedule of CNSs was spent respectively on telephone calls with other professionals and on telephone calls with patients and their families. This is an important finding as the time spent on phone calls is often overlooked in other studies of nursing care. The inclusion of this kind of specific information in existing dependency tools may help to improve their overall utility.

Interestingly, the perception of some of the interviewees in the current study that the CNS 
may be visiting patients too frequently and thereby keeping overall dependency levels too high was not supported by the quantitative findings, which showed that patient dependency levels tended to remain fairly stable over time. This disparity may have occurred owing to a misconception among a small number of the participants. It would be interesting in future work to explore the diagnosis of the patients in relation to overall dependency levels, particularly in light of developments in extending access to palliative care to patients with conditions such as heart failure, COPD, and dementia (Fisher, 2006; Yang et al, 2011).

The lack of any significant relationship between the two dependency tools and the total prioritization scores in this study may be related to the close proximity of each visit within the 3-month study period. A longer study period could be used in future work, especially given that the palliative care needs of patients with other conditions, such as heart failure and dementia, may fluctuate over time. Brady et al (2007) suggest that existing dependency tools should be adapted in line with the local context in order to reflect the differences in emphasis on certain aspects of care and the nurses' roles. Indeed, it was necessary in the present study to adapt the dependency tools used in order to reflect the additional factors that were considered important by the local CNS managers. However, these adaptations did not appear to significantly improve the tools. While such adaptation can affect the reliability and validity of an established instrument, none of these tools has as yet been subjected to a proper psychometric evaluation. Thus, there is a need for proper psychometric work and to examine how the incorporation of specific information relating to indirect nursing care and consultation with other health professionals might improve the status of current dependency tools. Other studies might also compare the short and longer-term outcomes for patients assessed with respect to dependency/complexity as well as the effects of patient dependency on the overall quality of nursing care.

Since the completion of this study, other research has also identified a gap in relation to the availability of successful tools to measure caseload dependency in community SPC, and recent attempts have been made to develop such tools (Dark et al, 2011). For example, other tools and programmes that were not available at the time of the study include the National End of Life Care Programme (NEoLCP, 2010), which aims to improve end-of-life care for adults through the implementation of the Department of Health's End of Life Care Strategy; the Hospice at Home Toolkit; and the Gold Standards Framework (GSF) Toolkit. The last of these, the GSF, has been developed recently in the UK for GPs and is designed to optimize patient care for those nearing the end of life. The GSF team are extending the use of this framework into many areas, including SPC. A key component of the GSF is the assessment of current and future clinical and personal needs. This toolkit may be useful in the future in terms of assisting in the measurement of patient dependency for CNSs working in SPC. There is a need for further research in this area in order to identify and evaluate one or more evidencebased tools for use in this setting. It might also be useful to encourage more collaborative work among palliative care teams in the UK and elsewhere in order to compare experiences of the use of dependency and prioritization tools in this and similar settings. For example, the Scottish Partnership for Palliative Care has undertaken interesting and relevant work in this area by supporting and contributing to the development and strategic direction of palliative care in Scotland (Scottish Partnership for Palliative Care, 2010).

\section{Conclusion}

This study was limited in size and scope, but was undertaken on an exploratory basis to address an important gap in our knowledge around the use of dependency tools in community-based SPC. The qualitative data were useful in helping to amplify some of the difficulties around introducing these kinds of tools into routine practice and in highlighting attendant workload concerns, all of which will prove useful to CNS managers in planning future service provision. The findings suggest that the Vale prioritization tool was most useful for the CNSs working in this area as opposed to tools that explicitly attempt to elicit information on different aspects/dimensions of dependency. In particular, the findings indicate that CNS managers in SPC should carefully consider the use of an appropriate tool that includes assessing the extent to which the patient lives alone and is in receipt of other services. However, further research is required to establish the applicability of these categories to other patient groups and to develop alternative tools for which reliability and validity can be properly tested. The two dependency tools in this study were also shown to have little predictive ability with regard to the amount of time CNSs spend in caring for patients. Thus, the utility of these tools to address

\section{It became evident ... that the participants felt under strain in their current occupational role. Further analysis is needed to establish whether this attitude is predominant within the service.?}


the workload concerns that also emerged from the findings is also questionable. It might be more useful and feasible for CNS managers to consider implementing a workload model and/or comprehensive review of the current workload demands placed on nurses in this regard. Lastly, the qualitative analysis highlighted further difficulties in using these kinds of tools in routine practice and much further research is required to better inform nursing practices in this area. IIPN

\section{Acknowledgments}

We would like to extend our thanks to all the nurses who agreed to take part in this study. We acknowledge with thanks the funding received for this project from a Health Research Board (Ireland) Summer Studentship and a Summer Programme for Undergraduate Research award from the National University of Ireland, Maynooth.

Ahmed N, Bestall JC, Ahmedzai SH, Payne SA, Clark D, Noble B (2004) Systematic review of the problems and issues of accessing specialist palliative care by patients, carers and health and social care professionals. Palliat Med 18(6): 525-42

Aitken AM (2009) Community Palliative Care: The Role of The Clinical Nurse Specialist. Blackwell Publishing, West Sussex, UK

Birch D, Fisher M, Grey A, Veitch J, Williams A (1997) The development of holistic dependency criteria for a specialist palliative care service. Int J Palliat Nurs 3(6): 306-16

Brady AM, Byrne G, Horan P, Griffiths C, MacGregor C, Begley C (2007) Measuring the workload of community nurses in Ireland: a review of workload measurement systems. J Nurs Manag 15(5): 481-9

Byrne G, Brady A, Griffith C, MacGregor C, Horan P, Begley C (2006) The Community Client Need Classification System - a dependency system for community nurses. J Nurs Manag 14(6): 437-46

Byrne G, Brady AM, Horan P, Macgregor C, Begley C (2007) Assessment of dependency levels of older people in the community and measurement of nursing workload. J Adv Nurs 60(1): 39-49

Crouch R, Williams S (2006) Patient dependency in the emergency department (ED): reliability and validity of the Jones Dependency Tool. Accid Emerg Nurs 14(4): 219-29

Dark D, Cardy C, Green S (2011) Prioritising care in a community palliative care setting using a dependency scoring tool. BMI Support Palliat Care 1: 218-9

Fadul N, Elsayem A, Palmer JL et al (2009) Supportive versus palliative care: what's in a name? A survey of medical oncologists and midlevel providers at a comprehensive cancer center. Cancer 115(9): 2013-21

Fisher K (2006) Specialist palliative care for patients with non-cancer diagnosis. Nurs Standard 21(4): 44-7

Gibbs J, McCoy A, Gibbs L, Rogers A, Addington-Hall J (2002) Living with and dying from heart failure: the role of palliative care. Heart 88(Suppl 2): 36-9

Grant M, Elk R, Ferrell B, Morrison RS, von Gunten CF (2009) Current status of palliative care-clinical implementation, education, and research. CA Cancer J Clin 59(5): 327-35

Graves J, Payne D (2007) The prioritisation of care in the Marie Curie nursing service. Marie Curie Cancer Care

Haines C, Perrott M, Weir P (2006) Promoting care for acutely ill children - development and evaluation of a paediatric early warning tool. Intensive Crit Care Nurs 22(2): 73-81
Hayes N (2000) Doing Psychological Research: Gathering and Analysing Data. Open University Press, Buckingham Hearn J, Higginson I (1998) Do specialist palliative care teams improve outcomes for cancer patients? A systematic literature review. Palliat Med 12(5): 317-32

Hirai K, Kudo T, Akiyama M et al (2011) Public awareness, knowledge of availability, and readiness for cancer palliative care services: a population-based survey across four regions in Japan. J Palliat Med 14(8): 918-22

Kolehmainen N, Francis J, Duncan E, Fraser C (2010) Community professionals' management of client care: a mixed-methods systematic review. J Health Serv Res Policy 15(1): 47-55

Lewis M, Pontin D (2008) Caseload management in community children's nursing. Paediatr Nurs 20(3): $18-22$

Manton KG, Corder LS, Stallard E (1993) Estimates of change in chronic disability and institutional incidence and prevalence rates in the U.S. elderly population from the 1982, 1984, and 1989 National Long Term Care Survey. J Gerontol 48(4): S153-S66

National End of Life Care Programme (2010) A framework of National Occupational Standards to support common core competences and principles for health and social care workers working with adults at the end of life. www.endoflifecareforadults.nhs.uk/assets/downloads/Core competences framework.pdf (accessed 12 December 2011)

Newbury J, De Leeuw W, Newton C (2008) What do community palliative care nurse specialists do? An activity analysis. Int J Palliat Nurs 14(6): 266-71

Newton J, Waters V (2001) Community palliative care clinical nurse specialists' descriptions of stress in their work. Int J Palliat Nurs 7(11): 531-40

Parker MG, Thorslund M (2007) Health trends in the elderly population: getting better and getting worse. Gerontologist 47(2): 150-8

Payne S, Smith P, Dean S (1999) Identifying the concerns of informal carers in palliative care. Palliat Med 13(1): $37-44$

Rauhala A, Kivimäki M, Fagerström L et al (2007) What degree of work overload is likely to cause increased sickness absenteeism among nurses? Evidence from the RAFAELA patient classification system. I Adv Nurs 57(3): 286-95

Rawlings D, Hendry K, Mylne S, Banfield M, Yates P (2011) Using palliative care assessment tools to influence and enhance clinical practice. Home Healthcare Nurs 29(3): 139-45

Schaffer M, Norlander L (2009) Being present: a nurse's resource for end-of-life care. SIGMA Theta Tau International: Indianapolis

Scottish Partnership for Palliative Care (2010) Annual Review for the year ended 31 March 2010. www.palliativecarescotland.org.uk/assets/files/annualReports/Annual\%20Review\%202010.pdf (accessed 12 December 2011)

Skilbeck J, Payne S (2003) Emotional support and the role of the clinical nurse specialists in palliative care. $J A d v$ Nurs 43(5): 521-30

Slade A, Fear J, Tennant A (2006) Identifying patients at risk of nursing home admission: the Leeds Elderly Assessment Dependency Screening Tool (LEADS). BMC Health Serv Res 6: 31-9

Sutherland J, Midgley C (2011) Extending services and enhancing systems: working together to manage increased numbers of referrals. BMJ Support Palliat Care 1: 206-73

Yang G, Ewing G, Booth S (2011) What is the role of specialist palliative care in an acute hospital setting? a qualitative study exploring views of patients and carers. Palliat Med [Epub ahead of print] doi:10.1177/0269216311425097

Yao CA, Hu WY, Lai YF, Cheng SY, Chen CY, Chiu TY (2007) Does dying at home influence the good death of terminal cancer patients? J Pain Symptom Manage 34(5): $497-504$ 
Copyright of International Journal of Palliative Nursing is the property of Mark Allen Publishing Ltd and its content may not be copied or emailed to multiple sites or posted to a listserv without the copyright holder's express written permission. However, users may print, download, or email articles for individual use. 